\title{
Note
}

\section{Postoperative changes in bone metabolism and bone mineral density in Japanese patients with acromegaly: a 3-year prospective study}

\author{
Daisuke Tamada $^{1)}$, Tetsuhiro Kitamura ${ }^{1)}$, Mitsuyoshi Takahara ${ }^{1)}$, Satoru Oshino ${ }^{2)}$, Youichi Saitoh ${ }^{2)}$, \\ Michio Otsuki ${ }^{1)}$ and Iichiro Shimomura ${ }^{1)}$ \\ 1) Department of Metabolic Medicine, Osaka University Graduate School of Medicine, Osaka 565-0871, Japan \\ 2) Department of Neurosurgery, Osaka University Graduate School of Medicine, Osaka 565-0871, Japan
}

\begin{abstract}
Growth hormone and insulin-like growth factor-I play important roles in regulating bone metabolism and bone mineral density in adulthood. However, the effect of excess growth hormone on bone metabolism and bone mineral density is not fully understood. Here, we investigated the long-term changes in bone metabolism and bone mineral density after a rapid decline in growth hormone levels due to transsphenoidal surgery in acromegalic patients. Eighteen acromegalic patients (10 males and 8 females) who underwent transsphenoidal surgery were enrolled in this prospective study. Bone formation marker (serum bone alkaline phosphatase), bone resorption marker (urinary type I collagen cross-linked $\mathrm{N}$-telopeptide), and bone mineral density were measured before surgery and at 3 months, 1 year, and 3 years after transsphenoidal surgery. While both serum bone alkaline phosphatase and urinary type I collagen cross-linked N-telopeptide levels decreased significantly after surgery, serum bone alkaline phosphatase/urinary type I collagen cross-linked $\mathrm{N}$-telopeptide ratio was significantly increased at 3 months and 3 years after surgery. Bone mineral density did not change markedly after surgery. In conclusion, the rapid decline in growth hormone levels following transsphenoidal surgery had no marked effect on bone mineral density for up to 3 years, despite significant changes in levels of bone turnover makers post-surgery.
\end{abstract}

Key words: Acromegaly, Bone metabolism, Bone mineral density, Transsphenoidal surgery

GROWTH HORMONE (GH) and insulin-like growth factor-I (IGF-I) are anabolic hormones for bone with important regulating effects on bone metabolism not only in childhood, but also in adulthood [1]. In active acromegaly, GH excess increases bone turnover, which is characterized predominantly by bone formation, resulting in an overall increase in BMD [2-4], although aging, sex, and hypogonadism in such patients is usually correlated with decreased BMD [3-5]. Although BMD in acromegalic patients should decrease after the treatment of transsphenoidal surgery (TSS), our previous study showed a rapid decline in bone turnover markers but not BMD in acromegalic patients at first

Submitted Mar. 25, 2015; Accepted Jul. 9, 2015 as EJ15-0174 Released online in J-STAGE as advance publication Jul. 28, 2015

Correspondence to: Michio Otsuki, M.D., Ph.D., Department of Metabolic Medicine, Osaka University Graduate School of Medicine, 2-2 Yamada-oka, Suita, Osaka 565-0871, Japan.

E-mail: otsuki@endmet.med.osaka-u.ac.jp
1 year after TSS [6]. While this observation is meaningful, the follow-up period of that study might not have been sufficient to evaluate BMD in acromegalic patients after TSS. In this study, we measured levels of bone turnover markers and BMD for 3 years after TSS in patients with active acromegaly.

\section{Subjects and Methods}

\section{Subjects}

This prospective study recruited 22 Japanese patients with untreated acromegaly who had been hospitalized at our department between April 2009 and May 2012. We excluded 4 patients for either receiving bisphosphonate treatment $(n=3)$ or reaching menopause during the study $(\mathrm{n}=1)$. A total of 18 Japanese patients (10 males and 8 females) with untreated acromegaly (median age: 52 years, range: $34-68$ years; median body mass index [BMI]: $24.1 \mathrm{~kg} / \mathrm{m}^{2}$, range: $20.8-30.9$ 
$\mathrm{kg} / \mathrm{m}^{2}$ ) were included in the final analysis (Table 1). The diagnosis of acromegaly and hypogonadism was described previously [6]. Hypoadrenalism was defined as a failed cortisol response (absolute value $<18 \mu \mathrm{g}$ / dL) to an insulin tolerance test (ITT). Hypothyroidism was defined as a free T4 level below the normal laboratory reference range (absolute value $<0.8 \mathrm{ng} / \mathrm{dL}$ ). GH deficiency (GHD) was not routinely assessed.

None of the patients had a history of bone fracture based on interviews, and none were taking any drugs to treat osteoporosis, including anti-osteoporosis drugs, vitamin $\mathrm{D}$, or calcium supplementation at the start of or throughout the study.

The study protocol was reviewed and approved by the ethics committee of Osaka University (reference no: 08116-5, UMIN no: 000004665) and performed in accordance with the Helsinki declaration. All patients provided informed consent before participation in the study.

\section{Methods}

All acromegaly patients underwent TSS. After TSS, serum GH levels were measured following a $75 \mathrm{~g}$ oral glucose tolerance test (OGTT). Patients in whom serum GH levels did not fall below $1 \mathrm{ng} / \mathrm{mL}$ after OGTT received pharmacological therapy with either or both long-acting agonists of somatostatin or dopamine agonists to control GH hypersecretion.

In all patients, GH, IGF-I, bone formation marker (serum bone alkaline phosphatase: BAP), bone resorption marker (urinary type I collagen cross-linked N-telopeptide: urinary NTx), and BMD were measured before surgery and at 3 months, 1 year, and 3 years after surgery as in our previous study [6].

\section{Statistical analysis}

The measured variables are expressed as median values (1st and 3rd quartiles). The time courses of all data were analyzed using generalized estimating equations (GEEs). Two-sided $P<0.05$ denoted a statistically significant difference. All statistical analyses were performed with R version 3.1.0 (R Core Team, Vienna, Austria).

\section{Results}

Eleven patients were considered to have reached remission, based on suppression of GH levels below $1 \mathrm{ng} / \mathrm{mL}$ after OGTT following TSS. GH hypersecretion in the remaining seven patients was not com-
Table 1 Clinical characteristics of acromegaly patients.

\begin{tabular}{|c|c|}
\hline $\mathrm{n}$ & 18 \\
\hline Men/Women & $10 / 8$ \\
\hline Age (years) & $52.0(43.5-60.8)$ \\
\hline BMI $\left(\mathrm{kg} / \mathrm{m}^{2}\right)$ & $24.1(22.6-25.4)$ \\
\hline Surgery alone (n, \%) & $11(61 \%)$ \\
\hline Surgery + medical therapy $(\mathrm{n}, \%)$ & $7(39 \%)$ \\
\hline Disease duration (years) & $8(2-20)$ \\
\hline $\mathrm{GH}(\mu \mathrm{g} / \mathrm{L})$ & $6.5(2.9-13.1)$ \\
\hline IGF-I $(\mu \mathrm{g} / \mathrm{L})$ & $593(394-758)$ \\
\hline IGF-I SD score & $7.0(4.8-8.4)$ \\
\hline Hypogonadism (n, \%) & $8(44 \%)$ \\
\hline Hypoadrenalism (n, \%) & $2(11 \%)$ \\
\hline Hypothyroidism (n, \%) & $3(17 \%)$ \\
\hline
\end{tabular}

BMI, body mass index; GH, growth hormone; IGF-I, insulin-like growth factor-I.

The measured variables are expressed as median values (1st and 3rd quartiles).

pletely controlled by TSS. Of these seven patients, three were treated with long-acting somatostatin analog, one received long-acting somatostatin analog and dopamine agonists, one received long-acting somatostatin analog and $\mathrm{GH}$ receptor antagonist, and two received dopamine agonists after TSS.

The characteristics of all acromegalic patients are summarized in Table 1. Eight patients were diagnosed with hypogonadism, two with hypoadrenalism, and three with diagnosed hypothyroidism at the beginning of this study. Hormone replacement therapy was performed in patients with hypoadrenalism and hypothyroidism; however, no therapy was conducted for those with hypogonadism. The hormone replacement therapy regimens described above were not altered during the study period.

GH and IGF-I levels were significantly decreased at 3 months, 1 year, and 3 years after TSS (Table 2). Serum IGF-1 levels were controlled in all patients to within age- and sex-adjusted reference ranges for a Japanese population [7]. While GHD was not routinely assessed, no patients had an IGF-I SD score less than -1 SD at 1 year, and only one patient was less than $-1 \mathrm{SD}$ at 3 years.

\section{Bone Metabolism}

\section{Baseline bone markers measurements}

At baseline, levels of bone formation marker (BAP, median: $24.5 \mu \mathrm{g} / \mathrm{L}$, range: $13.4-56.5 \mu \mathrm{g} / \mathrm{L}$; reference range: male 3.7-20.9 $\mu \mathrm{g} / \mathrm{L}$, female $2.9-14.5 \mu \mathrm{g} / \mathrm{L}$ ) and bone resorption marker (urinary NTx, median: $102 \mathrm{nmol} \cdot \mathrm{BCE} /$ $\mathrm{mmol} \cdot \mathrm{CRE}$, range: $34-354 \mathrm{nmol} \cdot \mathrm{BCE} / \mathrm{mmol} \cdot \mathrm{CRE}$; ref- 
Table 2 Postoperative changes in bone metabolism and BMD.

\begin{tabular}{lcccc}
\hline & Baseline & 3 months & 1 year & 3 year \\
\hline GH $(\mu \mathrm{g} / \mathrm{L})$ & $6.5(2.9-13.1)$ & $0.9(0.6-1.8)^{* *}$ & $0.7(0.4-1.2)^{* *}$ & $0.7(0.5-1.3)^{* *}$ \\
IGF-I $(\mu \mathrm{g} / \mathrm{L})$ & $593(394-758)$ & $199(161-320)^{* * *}$ & $186(162-243)^{* * *}$ & $158(131-221)^{* * *}$ \\
IGF-I S.D. score & $7.0(4.8-8.4)$ & $1.4(0.8-3.1)^{* * *}$ & $1.6(0.3-2.0)^{* * *}$ & $0.6(0.1-1.5)^{* * *}$ \\
BAP $(\mu \mathrm{g} / \mathrm{L})$ & $24.5(20.2-35.2)$ & $22.7(17.4-29.4)$ & $13.4(10.2-17.0)^{* * *}$ & $10.2(8.7-13.7)^{* * *}$ \\
Urinary NTx $(\mathrm{nmol} \cdot \mathrm{BCE} / \mathrm{mmol} \cdot \mathrm{CR})$ & $102(80-140)$ & $57(45-68)^{* *}$ & $41(33-58)^{* * *}$ & $31(18-58)^{* * *}$ \\
BAP/urinary NTx ratio & $0.25(0.13-0.62)$ & $0.44(0.16-1.17)^{* *}$ & $0.33(0.14-1.69)$ & $0.35(0.15-1.03)^{*}$ \\
Lumbar spine & & & \\
$\quad$ BMD $\left(\mathrm{g} / \mathrm{cm}^{2}\right)$ & $1.01(0.92-1.14)$ & $1.02(0.94-1.15)$ & $1.03(0.93-1.12)$ & $1.00(0.93-1.14)$ \\
T-score & $-0.2(-0.9-1.1)$ & $-0.2(-0.8-1.2)$ & $0.0(-0.8-0.9)$ & $-0.2(-0.8-0.8)$ \\
Z-score & $0.2(-0.1-1.4)$ & $0.3(0.1-1.6)$ & $0.3(0.0-1.3)$ & $0.4(-0.2-1.5)$ \\
Femoral neck & & & \\
BMD $\left(\mathrm{g} / \mathrm{cm}^{2}\right)$ & $0.77(0.72-0.85)$ & $0.77(0.70-0.80)$ & $0.79(0.71-0.86)$ & $0.79(0.69-0.87)$ \\
T-score & $-0.5(-0.8-0.3)$ & $-0.6(-0.8--0.1)$ & $-0.4(-0.7-0.3)$ & $-0.5(-1.0-0.5)$ \\
Z-score & $0.7(-0.4-1.3)$ & $0.6(-0.3-1.1)$ & $0.8(-0.1-1.1)$ & $0.9(-0.1-1.1)$ \\
33\% Radius & & & $0.65(0.56-0.74)$ & $0.64(0.57-0.73)$ \\
BMD $\left(\mathrm{g} / \mathrm{cm}^{2}\right)$ & $0.65(0.56-0.74)$ & $0.66(0.56-0.76)$ & $-0.5(-2.7-0.9)$ & $-0.6(-2.5-0.6)$ \\
T-score & $-1.0(-2.6-1.0)$ & $-0.8(-2.7-1.2)$ & $0.4(-1.8-1.5)$ & $0.5(-1.5-1.5)$ \\
Z-score & $0.0(-1.7-1.6)$ & $0.3(-1.8-1.9)$ &
\end{tabular}

GH, growth hormone; IGF-I, insulin-like growth factor-I; BAP, serum bone alkaline phosphatase; urinary NTx, urinary type I collagen cross-linked N-telopeptide; BMD, bone mineral density.

Comparison vs. baseline. $* P<0.05, * * P<0.01, * * * P<0.001$

The measured variables are expressed as median values (1st and 3 rd quartiles).

erence range: $9.3-54.3 \mathrm{nmol} \cdot \mathrm{BCE} / \mathrm{mmol} \cdot \mathrm{CRE}$ ) in acromegalic patients were high before TSS compared with respective reference ranges (Table 2). The frequency of abnormal BAP elevation was $83 \%$ (men: $70 \%$, women: $100 \%$ ), and the frequency of abnormal urinary NTx elevation was $83 \%$ (men: $90 \%$, women: $75 \%$ ).

\section{Longitudinal bone marker measurements after 3 years of follow-up}

BAP was significantly decreased at 1 and 3 years after TSS, but not at 3 months, compared with the baseline (Table 2). In contrast, urinary NTx was significantly decreased at all follow-up points after TSS compared with the baseline (Table 2). These results demonstrated high levels of bone formation and resorption in acromegalic patients before TSS, with both decreasing after TSS. Further, the decrease in bone resorption occurred earlier than that of bone formation after TSS. BAP/urinary NTx ratio was significantly increased at 3 months and 3 years after TSS compared with the baseline value (Table 2). The decline of bone turnover following TSS was bone resorption dominantly.

\section{BMD measurements}

\section{Baseline BMD measurements}

At baseline, median BMD at the lumbar spine was
$1.01 \mathrm{~g} / \mathrm{cm}^{2}$ (range: $0.92-1.14 \mathrm{~g} / \mathrm{cm}^{2}$ ), median T-score was -0.2 (range: $-0.9-1.1$ ), and median Z-score was 0.2 (range: $-0.1-1.4$ ) (Table 2). Median BMD at the femoral neck was $0.77 \mathrm{~g} / \mathrm{cm}^{2}$ (range: $0.72-0.85 \mathrm{~g} / \mathrm{cm}^{2}$ ), median T-score was -0.5 (range: $-0.8-0.3$ ), and median Z-score was 0.7 (range: -0.1-1.4) (Table 2). Median $\mathrm{BMD}$ at the $33 \%$ radius was $0.65 \mathrm{~g} / \mathrm{cm}^{2}$ (range: 0.56 $0.74 \mathrm{~g} / \mathrm{cm}^{2}$ ), median T-score was -1.0 (range: -2.6 1.0 ), and median Z-score was 0.0 (range: $-1.7-1.6$ ) (Table 2). No patients had osteoporosis at the lumbar spine or femoral neck at baseline, but $4(22 \%)$ had osteopenia. Six patients (33\%) had osteoporosis at the $33 \%$ radius at baseline, and $3(17 \%)$ had osteopenia.

\section{Longitudinal BMD measurements after 3 years of follow-up}

GEE showed no decrease in BMD at 3 months, 1 year, or 3 years after TSS at any measured bone sites (Fig. 1 A-C). No significant changes were noted in male or female patients in BMD throughout the study (Fig. 1 D-I), nor were any differences in percentchange in BMD found after TSS, relative to pre-surgery values. Of note, neither the presence of hypogonadism nor BMD level at baseline had any influence on changes in BMD during the study (data not shown). In order to investigate the effect of decline in GH levels 

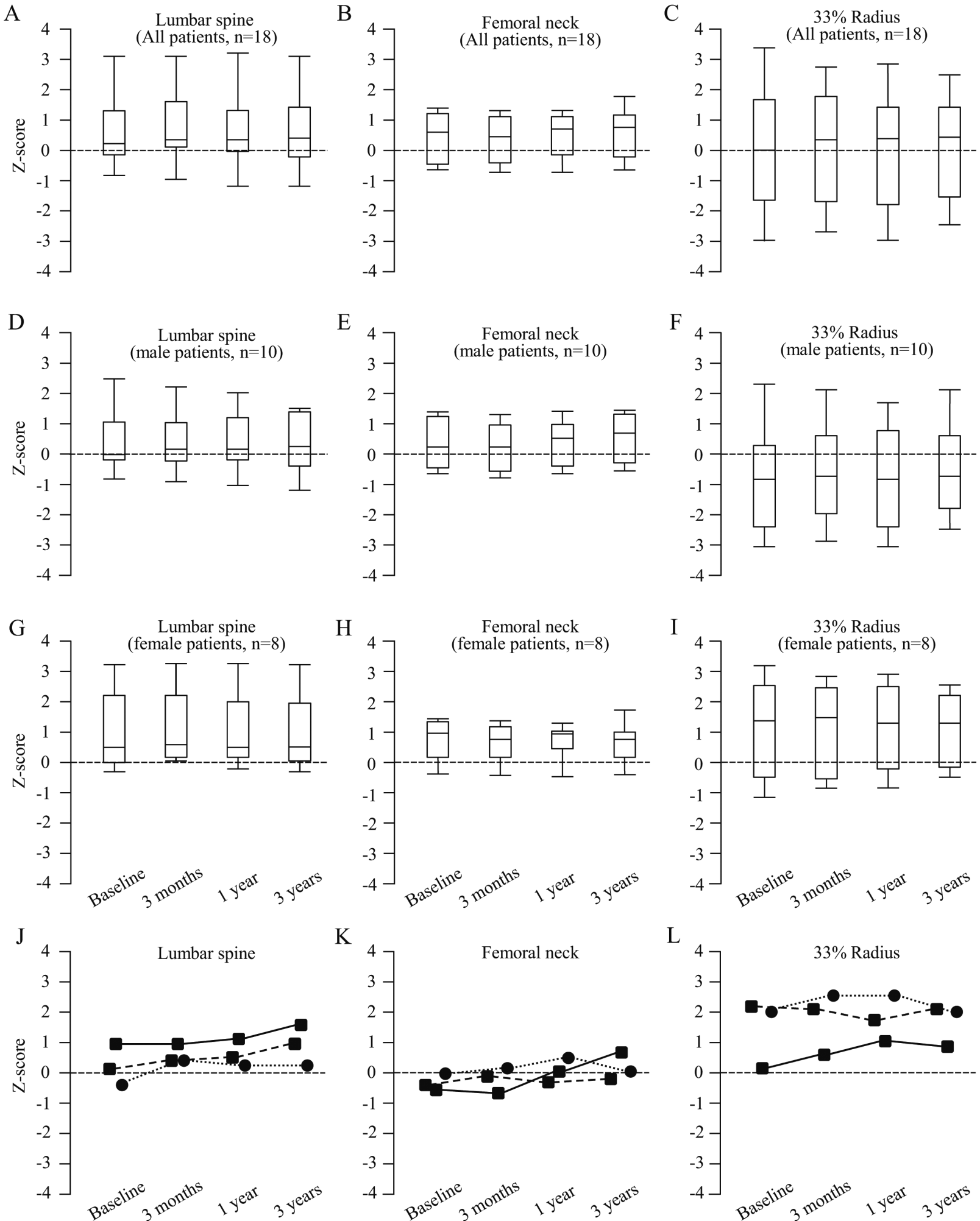

Fig. 1 Change in bone mineral density (BMD) after transsphenoidal surgery (TSS) (Z-score).

BMD of all patients $(n=18, A, B, C)$, male $(n=10, D, E, F)$ and female $(n=8, G, H, I)$ patients was not decreased at 3 months or 1 or 3 years after TSS. BMD of three patients ( 2 males; - and 1 female; $\bullet$ ) who satisfies the following criteria: 1$)$ under 45 years old, 2) reached remission by TSS only (serum GH levels below $0.4 \mathrm{ng} / \mathrm{mL}$ after a $75 \mathrm{~g}$ oral glucose tolerance test (OGTT) with normal serum IGF-I levels (IGF-I S.D. score < 1.5)), and 3) not required hormone replacement therapy after TSS (J, K, L). 
after TSS excluding subject heterogeneity, we selected the BMD of three patients ( 2 males and 1 female) who satisfies the following criteria: 1) under 45 years old, 2) remission by TSS by itself (serum GH levels below $0.4 \mathrm{ng} / \mathrm{mL}$ after an OGTT with normal serum IGF-I levels (IGF-I S.D. score $<1.5)$ ), and 3) not required hormone replacement therapy after TSS (Fig. $1 \mathrm{~J}-\mathrm{L}$ ). Their BMD at the lumber spine and the femoral neck increased at the 3 years after TSS. On the other hand, the $\mathrm{BMD}$ at the $33 \%$ radius was not changed.

\section{Discussion}

In the present study, treatment of acromegalic patients with TSS significantly reduced bone turnover over the long term. However, BMD was not decreased in the 3 years after TSS.

We previously reported changes in levels of bone turnover markers and BMD in acromegalic patients in the first year following TSS [6]. In the present study, BMD remained stable up to three years after TSS, despite significant changes in levels of bone turnover makers. The results of BMD are explained by changes in bone metabolism, with reduction of bone resorption occurring much earlier than bone formation due to a post-surgery decline in GH levels. Further, in the current study, BAP/urinary NTx ratio was significantly increased at 3 months and 3 years after TSS. Previous studies have reported that the ratio of procollagen type I N-propeptide (PINP) and C-terminal cross-linking telopeptide (CTX-I), other bone turnover markers, reflects the bone remodeling balance [8]. The increase of BAP/urinary NTx ratio after TSS in acromegalic patients suggests that bone formation is superior to bone resorption. The discrepancy in this bone remodeling balance may explain the maintenance of BMD after TSS in acromegalic patients. We also found that BMD at the lumber spine and the femoral neck increased at the 3 years after TSS in only three acromegalic patients whose condition were suitable for the change of GH levels after TSS.

Recently, Claessen et al. reported that BMD at the lumbar spine and total hip did not change markedly in 15 acromegalic patients in remission for 31 months after TSS [9]. Further, they noted no marked differences in BMD between men and women. These authors' results seem consistent with our present findings; however, of note, their acromegalic patients had been in remission for an average of 18.1 years (range: $11-28$ years) at the beginning of their study. Their study point might therefore have been too late to evaluate the effect of $\mathrm{GH}$ decline due to treatment for acromegaly on BMD. Our study has the distinct advantage of having started evaluation of BMD before TSS.

Mazziotti et al. reported that BMD decreased significantly at the femoral neck but did not change at the lumbar spine in acromegalic patients after 3 years of follow-up [10]. Their results regarding BMD at the femoral neck differ from ours, likely because the frequency of hormone replacement for hypopituitarism in their patients was much higher than in our patients (replacement for hypoadrenalism, 56\% vs. $11 \%$; replacement for hypothyroidism, $52 \%$ vs. $17 \%$ ). Glucocorticoid replacement was independently associated with reduced BMD at the femoral neck and lumber spine and an increased frequency of osteopenia in women with ACTH insufficiency [11]. In primary hypothyroidism patients, supraphysiological doses of L-thyroxine decreased BMD at the femoral neck and lumber spine [12]. These previous findings suggest that hormone replacement for hypoadrenalism and hypothyroidism may reduce BMD. Alternatively, difference in number or quality of cured acromegaly between previous reports and current study may explain.

The risk of bone fracture is still unclear in active acromegalic patients [13-15] as well as after treatment $[9,10]$. However, whether or not maintenance of BMD after TSS reduced risk of fracture is unclear, as we were unable to evaluate the risk of bone fracture in the current study. Further, we did not evaluate bone quality. Clearly, a study about bone quality and a prospective study to evaluate the incidence of bone fractures in a large number of acromegalic patients will be needed to clarify these points.

In conclusion, the present study demonstrated that a rapid decline in GH levels after TSS does not affect BMD up to 3 years after surgery in a limited number of heterogenous patients with treated acromegaly, despite significant changes in levels of bone turnover markers.

\section{Disclosures}

The authors declare no personal financial or institutional interest in this article. 


\section{References}

1. Giustina A, Mazziotti G, Canalis E (2008) Growth hormone, insulin-like growth factors, and the skeleton. Endocr Rev 29: 535-559.

2. Ueland $\mathrm{T}$, Fougner SL, Godang $\mathrm{K}$, Schreiner $\mathrm{T}$, Bollerslev J (2006) Serum GH and IGF-I are significant determinants of bone turnover but not bone mineral density in active acromegaly: a prospective study of more than 70 consecutive patients. Eur J Endocrinol 155: 709-715.

3. Zgliczynski W, Kochman M, Misiorowski W, Zdunowski P (2007) In acromegaly, increased bone mineral density (BMD) is determined by GH-excess, gonadal function and gender. Neuro Endocrinol Lett 28: 621-628.

4. Bolanowski M, Daroszewski J, Medraś M, ZadroznaSliwka B (2006) Bone mineral density and turnover in patients with acromegaly in relation to sex, disease activity, and gonadal function. J Bone Miner Metab 24: 72-78.

5. Kaji H, Sugimoto T, Nakaoka D, Okimura Y, Kaji H, et al. (2001) Bone metabolism and body composition in Japanese patients with active acromegaly. Clin Endocrinol $(O x f)$ 55: 175-181.

6. Tamada D, Kitamura T, Onodera T, Tabuchi Y, Fukuhara A, et al. (2014) Rapid decline in bone turnover markers but not bone mineral density in acromegalic patients after transsphenoidal surgery. Endocr J 61: 231-237.

7. Isojima T, Shimatsu A, Yokoya S, Chihara K, Tanaka T, et al. (2012) Standardized centile curves and reference intervals of serum insulin-like growth factor-I (IGF-I) levels in a normal Japanese population using the LMS method. Endocr J 59: 771-780.

8. Behan LA, Kelleher G, Hannon MJ, Brady JJ, Rogers B, et al. (2013) Low-dose hydrocortisone replace- ment therapy is associated with improved bone remodelling balance in hypopituitary male patients. Eur $J$ Endocrinol 170: 141-150.

9. Claessen KM, Kroon HM, Pereira AM, AppelmanDijkstra NM, Verstegen MJ, et al. (2013) Progression of vertebral fractures despite long-term biochemical control of acromegaly: a prospective follow-up study. $J$ Clin Endocrinol Metab 98: 4808-4815.

10. Mazziotti G, Bianchi A, Porcelli T, Mormando M, Maffezzoni F, et al. (2013) Vertebral fractures in patients with acromegaly: a 3 -year prospective study. $J$ Clin Endocrinol Metab 98: 3402-3410.

11. Ragnarsson O, Nystrom HF, Johannsson G (2012) Glucocorticoid replacement therapy is independently associated with reduced bone mineral density in women with hypopituitarism. Clin Endocrinol (Oxf) 76: 246252.

12. De Rosa G, Testa A, Giacomini D, Carrozza C, Astazi P, et al. (1997) Prospective study of bone loss in preand post-menopausal women on L-thyroxine therapy for non-toxic goitre. Clin Endocrinol (Oxf) 47: 529-535.

13. Mazziotti G, Bianchi A, Bonadonna S, Cimino V, Patelli I, et al. (2008) Prevalence of vertebral fractures in men with acromegaly. J Clin Endocrinol Metab 93: 46494655 .

14. Bonadonna S, Mazziotti G, Nuzzo M, Bianchi A, Fusco A, et al. (2005) Increased prevalence of radiological spinal deformities in active acromegaly: a cross-sectional study in postmenopausal women. J Bone Miner Res 20: 1837-1844.

15. Vestergaard P, Mosekilde L (2004) Fracture risk is decreased in acromegaly--a potential beneficial effect of growth hormone. Osteoporos Int 15: 155-159. 